See discussions, stats, and author profiles for this publication at: https://www.researchgate.net/publication/315907114

\title{
Nonsymmetric tensor description of massive spin-2 particles in a curved background
}

Article $\cdot$ March 2017

DOI: 10.1103/PhysRevD.95.065028

CITATIONS

3

2 authors:

\section{Dalmazi}

São Paulo State University

76 PUBLICATIONS 418 CITATIONS

SEE PROFILE

Some of the authors of this publication are also working on these related projects:
READS

31

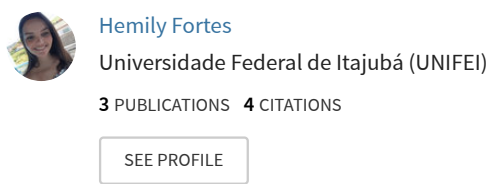

Project spin-3 in $D=2+1$ View project 
PHYSICAL REVIEW D 95, 065028 (2017)

\title{
Nonsymmetric tensor description of massive spin-2 particles in a curved background
}

\author{
D. Dalmazi* and H. G. M. Fortes ${ }^{\dagger}$ \\ UNESP-Campus de Guaratinguetá-DFQ Avenida Dr. Ariberto Pereira da Cunha, \\ 333 CEP 12516-410 Guaratinguetá, São Paulo, Brazil \\ (Received 5 February 2017; published 30 March 2017)
}

\begin{abstract}
Massive spin-2 particles have been a subject of great interest in current research. If the graviton has a small mass, the gravitational force at large distances decreases more rapidly, which could contribute to the explanation of the accelerated expansion of the Universe. The massive spin-2 particles are commonly described by the known Fierz-Pauli action which is formulated in terms of a symmetric tensor $h_{\mu \nu}=h_{\nu \mu}$. However, the Fierz-Pauli theory is not the only possible description of massive spin-2 particles via a rank-2 tensor. There are other two families of models $\mathcal{L}\left(a_{1}\right)$ and $\mathcal{L}_{n F P}(c)$, where $a_{1}$ and $c$ are real arbitrary parameters, which describe massive particles of spin-2 in the flat space via a nonsymmetric tensor $e_{\mu \nu} \neq e_{\nu \mu}$. In the present work we derive Lagrangian constraints stemming from $\mathcal{L}\left(a_{1}\right)$ and $\mathcal{L}_{n F P}(c)$ in curved backgrounds with nonminimal couplings which are analytic functions of $\mathrm{m}^{2}$. We show that the constraints lead to a correct counting of degrees of freedom if nonminimal terms are included with fine-tuned coefficients and the background space is of the Einstein type, very much like the Fierz-Pauli case. We also examine the existence of local symmetries.
\end{abstract}

DOI: $10.1103 /$ PhysRevD.95.065028

\section{INTRODUCTION}

Our motivation to work with massive spin-2 particles in a curved background is twofold. On one hand, they can represent massive gravitons at the linearized approximation; on the other hand, they can be understood as elementary massive spin- 2 particles in a given gravitational background.

Regarding the motivation for massive gravitons, they lead to a weaker gravitational interaction at large distances, which could contribute to the observed [1,2] accelerated expansion of the Universe at large distances. Although the recent detection of gravitational waves [3] is consistent with massless gravitons, predicted by the usual (massless) general relativity, massive gravitons are not ruled out. The mentioned experiment sets an upper bound of about $10^{-22} \mathrm{eV}$ for the graviton mass [4]. Furthermore, previous theoretical obstacles for massive gravitons like the vDVZ mass discontinuity $[5,6]$ and the existence of ghosts in the nonlinear theory [7] have been tackled by the addition of fine-tuned nonlinear self-interaction terms for the metric fluctuation; see [8] and the bimetric model of [9]. Those models are based on previous ideas of [10] and [11] and have recently led to intense work on massive gravity and related topics; see the review works $[12,13]$.

Regarding elementary massive spin-2 particles, the coupling of higher spin particles to electromagnetic and

\footnotetext{
*dalmazi@feg.unesp.br

†hemily.gomes@gmail.com
}

gravitational interactions is a longstanding problem. Since any elementary particle must couple to gravity, one first needs to check the gravitational interaction as in $[14,15]$ and [16]. Usually, unitarity [7] and causality [17,18] are lost in interacting theories of higher spin particles. Those particles require the use of higher rank tensors which have too many components. The redundant components must vanish on shell. They work like auxiliary fields. However, when interactions are turned on, some of those auxiliary fields may become dynamic, giving rise to negative contributions to the Hamiltonian (instabilities) and incorrect number of degrees of freedom.

Basically all studies of interacting massive spin-2 particles and the modern massive gravity theories, as [8], start with the paradigmatic free theory suggested by Fierz and Pauli in [19]. It describes massive spin-2 particles via a symmetric and traceful rank-2 tensor $h_{\mu \nu}=h_{\nu \mu}$. It is the metric fluctuation in massive gravitational theories, $g_{\mu \nu}=\eta_{\mu \nu}+h_{\mu \nu}$. A natural question concerns the independence of the outcome of such studies on the underlying specific massive spin-2 model.

In [20] we started with a rather general second order (in derivatives) Ansatz for a quadratic Lagrangian for a nonsymmetric rank-2 tensor $e_{\mu \nu}$ and by requiring the existence of only one massive physical pole in the spin-2 sector of the propagator we obtained three families of consistent free theories describing massive spin-2 particles. One of them is the usual Fierz-Pauli (FP) family which includes the FP model written in terms of a symmetric tensor. The other two families require a nonsymmetric tensor. There is no local field redefinition 
relating those families in general. One of the families is given in (1) and the other one in (49). They depend on an arbitrary real constant, $a_{1}$ and $c$, respectively. See also [21] for the special case $a_{1}=-1 / 4$. Here we couple a background gravitational field to those theories by including also nonminimal terms and look for curved space generalization of the tensor, vector, and scalar constraints which are necessary for getting rid of nonphysical degrees of freedom. We require that the coefficients of the nonmininal terms be analytic functions of $\mathrm{m}^{2}$. Such a restriction plays a key role in our work and leads us to constrain the gravitational background to Einstein spaces; see further comments in the conclusion. In Sec. II we deal with $\mathcal{L}\left(a_{1}\right)$, while in Sec. III we study the $\mathcal{L}_{n F P}(c)$ case. In Sec. IV we draw our conclusions. In the Appendix we briefly show the technical difficulties in arbitrary backgrounds.

\section{FAMILY OF LAGRANGANS $\mathcal{L}\left(a_{1}\right)$}

\section{A. Main results in the flat space}

In [22] the family of second order Lagrangians $\mathcal{L}\left(a_{1}\right)$ has been presented in arbitrary dimensions $D \geq 3$, but here we focus on $D=4$. It describes massive "spin-2" particles via a nonsymmetric rank-2 tensor $e_{\mu \nu} \neq e_{\nu \mu}$ in the flat space ${ }^{1}$ for any value of the constant $a_{1}$ :

$$
\begin{aligned}
\mathcal{L}\left(a_{1}\right)= & -\frac{1}{2} \partial^{\mu} e^{(\alpha \beta)} \partial_{\mu} e_{(\alpha \beta)} \\
& +\left(a_{1}+\frac{1}{4}\right) \partial^{\mu} e\left[\partial_{\mu} e-2 \partial^{\alpha} e_{(\alpha \mu)}\right] \\
& +\left[\partial^{\alpha} e_{(\alpha \beta)}\right]^{2}+\left(a_{1}-\frac{1}{4}\right)\left(\partial^{\alpha} e_{\alpha \beta}\right)^{2} \\
& -\frac{m^{2}}{2}\left(e_{\mu \nu} e^{\nu \mu}-e^{2}\right) .
\end{aligned}
$$

We recover the FP theory at $a_{1}=1 / 4$ where $e_{[\mu \nu]}$ becomes nondynamic and it can be neglected. However there is no local field redefinition which takes us from the FP theory to $a_{1} \neq 1 / 4$. The massless theory $\mathcal{L}_{m=0}\left(a_{1}\right)$ is unitary in the ranges $a_{1} \geq 1 / 4$ and $a_{1} \leq-1 / 12$; it describes massless spin-2 particles plus a scalar field, except at $a_{1}=1 / 4$ and $a_{1}=-1 / 12$ where the scalar field disappears. At $a_{1}=-1 / 12$ the model $\mathcal{L}\left(a_{1}\right)$ intersects the nFP (non-Fierz-Pauli) family of Sec. III at $c=-1$; see (49).

The flat space equations of motion $E_{\mu \nu} \equiv \frac{\delta S\left(a_{1}\right)}{\delta e^{\mu \nu}}=0$ are given by

\footnotetext{
${ }^{1}$ Throughout this work we use $\eta_{\mu \nu}=(-,+,+,+), e_{(\alpha \beta)}=$ $\left(e_{\alpha \beta}+e_{\beta \alpha}\right) / 2$ and $e_{[\alpha \beta]}=\left(e_{\alpha \beta}-e_{\beta \alpha}\right) / 2$.
}

$$
\begin{aligned}
E_{\mu \nu}= & \square e_{(\mu \nu)}+2\left(a_{1}+\frac{1}{4}\right)\left[\eta_{\mu \nu}\left(\partial^{\alpha} \partial^{\beta} e_{\alpha \beta}-\square e\right)+\partial_{\mu} \partial_{\nu} e\right] \\
& -\partial_{\mu} \partial^{\alpha} e_{(\alpha \nu)}+-\partial_{\nu} \partial^{\alpha} e_{(\alpha \mu)}-2\left(a_{1}-\frac{1}{4}\right) \partial_{\mu} \partial^{\alpha} e_{\alpha \nu} \\
& +m^{2}\left(\eta_{\mu \nu} e-e_{\nu \mu}\right)=0 .
\end{aligned}
$$

From $\partial^{\nu} E_{\mu \nu}=0$, we have the vector constraint:

$$
\partial^{\alpha} e_{\alpha \nu}=\partial_{\nu} e .
$$

Plugging (3) back in $E_{\mu \nu}$ we have, from $E_{\mu \nu}-E_{\nu \mu}=0$, the tensor constraint:

$$
e_{[\mu \nu]}=0 .
$$

From (3) and (4) back in $\eta^{\mu \nu} E_{\mu \nu}=0$, we obtain the final scalar constraint:

$$
e=0
$$

and, consequently, from (3) we have the transverse condition:

$$
\partial^{\alpha} e_{\alpha \nu}=0
$$

The equations of motion $E_{\mu \nu}=0$ become the KleinGordon equations

$$
\left(\square-m^{2}\right) e_{(\mu \nu)}=0
$$

The FP conditions (4), (5) and (6) guarantee the correct number of 5 degrees of freedom consistent with $5=2 s+1$, see [23] for a recent derivation of the FP conditions from first principles.

\section{B. Generalization of $\mathcal{L}\left(a_{1}\right)$ to curved spaces}

\section{General setup and constraints}

If we want to construct a theory of massive spin-2 field in a curved space out of a nonsymmetric rank-2 tensor we should provide the same number of propagating degrees of freedom as in the flat case. They correspond to the curved space version of the 11 Fierz-Pauli conditions (4), (5), (6), namely $e_{[\mu \nu]}=0, g^{\mu \nu} e_{\mu \nu}=0$ and $\nabla^{\mu} e_{\mu \nu}=0$. Thus, from the 16 components of $e_{\mu \nu}$, we end up with $16-11=5$ degrees of freedom. Our calculations focus on the $D=4$ case, but it can be generalized to $D$ dimensions $(D \geq 3)$. 
Generalizing (1) to curved spacetime we substitute all derivatives by the covariant ones and add nonminimal terms containing the curvature tensor as in the FP case [24]. They also take care of ordering ambiguities. Requiring a quadratic theory in derivatives, consistent with the flat limit (1) and at most linear in curvatures, the most general action has the form ${ }^{2}$

$$
\begin{aligned}
\mathcal{L}^{g}\left(a_{1}\right)= & -\frac{1}{4} \nabla^{\mu} e^{\alpha \beta} \nabla_{\mu} e_{\alpha \beta}-\frac{1}{4} \nabla^{\mu} e^{\alpha \beta} \nabla_{\mu} e_{\beta \alpha}+a_{1} \nabla^{\alpha} e_{\alpha \beta} \nabla_{\mu} e^{\mu \beta}+\frac{1}{2} \nabla^{\alpha} e_{\alpha \beta} \nabla_{\mu} e^{\beta \mu}+\frac{1}{4} \nabla^{\alpha} e_{\beta \alpha} \nabla_{\mu} e^{\beta \mu}+\left(a_{1}+\frac{1}{4}\right) \nabla^{\mu} e \nabla_{\mu} e \\
& -\left(a_{1}+\frac{1}{4}\right) \nabla^{\mu} e \nabla^{\alpha} e_{\alpha \mu}+-\left(a_{1}+\frac{1}{4}\right) \nabla^{\mu} e \nabla^{\alpha} e_{\mu \alpha}-\frac{m^{2}}{2}\left(e_{\alpha \beta} e^{\beta \alpha}-e^{2}\right)+f_{1} R e^{\alpha \beta} e_{\alpha \beta}+f_{2} R e^{2} \\
& +f_{3} R_{\alpha \beta \mu \nu} e^{\alpha \mu} e^{\beta \nu}+f_{4} R_{\alpha \beta} e^{\alpha \mu} e^{\beta}{ }_{\mu}+f_{5} R_{\alpha \beta} e^{\alpha \beta} e+f_{6} R_{\alpha \beta \mu \nu} e^{\alpha \beta} e^{\mu \nu}+f_{7} R_{\alpha \beta} e^{\alpha \mu} e_{\mu}{ }^{\beta}+f_{8} R e^{\alpha \beta} e_{\beta \alpha}+f_{9} R_{\alpha \beta} e^{\mu \alpha} e_{\mu}{ }^{\beta}
\end{aligned}
$$

where $f_{j}(j=1,2, \ldots, 9)$ are arbitrary constants for the time being.

Varying the action with respect to $e^{\rho \sigma}$, we obtain the equations of motion in curved space:

$$
\begin{aligned}
E_{\rho \sigma} \doteq \frac{\delta S}{\delta e^{\rho \sigma}}= & \frac{1}{2} \square\left(e_{\rho \sigma}+e_{\sigma \rho}\right)-2 a_{1} \nabla_{\rho} \nabla^{\mu} e_{\mu \sigma}-\frac{1}{2} \nabla_{\rho} \nabla^{\mu} e_{\sigma \mu}-\frac{1}{2} \nabla_{\sigma} \nabla^{\mu} e_{\mu \rho}-\frac{1}{2} \nabla_{\sigma} \nabla^{\mu} e_{\rho \mu} \\
& +2\left(a_{1}+\frac{1}{4}\right)\left[-g_{\rho \sigma} \square e+\nabla_{\rho} \nabla_{\sigma} e+g_{\rho \sigma} \frac{\nabla^{\mu} \nabla^{\alpha}\left(e_{\alpha \mu}+e_{\mu \alpha}\right)}{2}\right]+-m^{2}\left(e_{\sigma \rho}-e g_{\rho \sigma}\right)+2 f_{1} R e_{\rho \sigma} \\
& +2 f_{2} R g_{\rho \sigma} e+2 f_{3} R_{\rho \beta \sigma \nu} e^{\beta \nu}+2 f_{4} R_{\rho \beta} e^{\beta}{ }_{\sigma}+f_{5} R_{\rho \sigma} e+f_{5} R_{\alpha \beta} g_{\rho \sigma} e^{\alpha \beta}+2 f_{6} R_{\alpha \beta \rho \sigma} e^{\alpha \beta}+f_{7} R_{\alpha \sigma} e^{\alpha} \\
& +f_{7} R_{\rho \alpha} e_{\sigma}^{\alpha}+2 f_{8} R e_{\sigma \rho}+2 f_{9} R_{\sigma \beta} e_{\rho}^{\beta}=0 .
\end{aligned}
$$

By applying one derivative on the equations of motion and after several manipulations, one obtains:

$$
\begin{aligned}
\mathcal{C}_{\rho} \doteq \nabla^{\sigma} E_{\rho \sigma}= & +\left(1-2 f_{3}-2 f_{6}\right) R_{\rho \lambda \sigma \alpha} \nabla^{\alpha} e^{\lambda \sigma}+\left(1+2 f_{6}\right) R_{\rho \lambda \sigma \alpha} \nabla^{\alpha} e^{\sigma \lambda}+\left(\frac{1}{2}+f_{7}\right) R^{\lambda \alpha} \nabla_{\alpha} e_{\lambda \rho} \\
& +\left(\frac{1}{2}+2 f_{9}\right) R^{\lambda \alpha} \nabla_{\alpha} e_{\rho \lambda}+\left(f_{7}-2 a_{1}\right) R_{\lambda \rho} \nabla_{\mu} e^{\mu \lambda}+\left(2 f_{4}-\frac{1}{2}\right) R_{\lambda \rho} \nabla_{\mu} e^{\lambda \mu}+2 f_{1} R \nabla^{\sigma} e_{\rho \sigma} \\
& +\left(\frac{1}{4}+2 f_{1}+f_{9}\right) e_{\rho \sigma} \nabla^{\sigma} R+\left(2 f_{2}+\frac{f_{5}}{2}\right) e \nabla_{\rho} R+\left(m^{2}+2 f_{2} R\right) \nabla_{\rho} e+\left(\frac{1}{2}+2 f_{4}+2 f_{6}\right) e^{\beta \sigma} \nabla_{\sigma} R_{\rho \beta} \\
& +\left(\frac{1}{2}+2 a_{1}+f_{5}\right) R_{\rho \sigma} \nabla^{\sigma} e-\left(1-2 f_{3}-f_{5}\right) e^{\alpha \beta} \nabla_{\rho} R_{\alpha \beta}+f_{5} R_{\alpha \beta} \nabla_{\rho} e^{\alpha \beta}+ \\
& -\left(\frac{1}{2}+2 f_{3}+2 f_{6}-f_{7}\right) e^{\sigma \alpha} \nabla_{\sigma} R_{\rho \alpha}+\left(\frac{1}{4}+\frac{f_{7}}{2}+2 f_{8}\right) e_{\sigma \rho} \nabla^{\sigma} R+-\left(m^{2}-2 f_{8} R\right) \nabla^{\sigma} e_{\sigma \rho} .
\end{aligned}
$$

Now, we define the tensor $\mathcal{C}_{\rho \sigma}$ :

$$
\begin{aligned}
\mathcal{C}_{\rho \sigma} \doteq E_{\rho \sigma}-E_{\sigma \rho}= & \left(-2 a_{1}+\frac{1}{2}\right)\left(\nabla_{\rho} \nabla^{\mu} e_{\mu \sigma}-\nabla_{\sigma} \nabla^{\mu} e_{\mu \rho}\right)+\left[m^{2}+2 R\left(f_{1}-f_{8}\right)\right]\left(e_{\rho \sigma}-e_{\sigma \rho}\right)+2 f_{3} R_{\rho \beta \sigma \nu}\left(e^{\beta \nu}-e^{\nu \beta}\right) \\
& +2 f_{4}\left(R_{\rho \beta} e^{\beta}{ }_{\sigma}-R_{\sigma \beta} e^{\beta}{ }_{\rho}\right)+4 f_{6} R_{\alpha \beta \rho \sigma} e^{\alpha \beta}+f_{7} R_{\sigma}^{\alpha}\left(e_{\alpha \rho}-e_{\rho \alpha}\right) \\
& +f_{7} R_{\rho}{ }^{\alpha}\left(e_{\sigma \alpha}-e_{\alpha \sigma}\right)+2 f_{9}\left(R_{\sigma \beta} e_{\rho}{ }^{\beta}-R_{\rho \beta} e_{\sigma}^{\beta}\right) .
\end{aligned}
$$

In order to find a scalar constraint we have to consider the most general scalar combination of the equations of motion

$$
\mathcal{C} \doteq\left(b_{0} R+b_{1} m^{2}\right) g^{\rho \sigma} E_{\rho \sigma}+b_{2} R^{\rho \sigma} E_{\rho \sigma}+b_{3} \nabla^{\rho} \nabla^{\sigma} E_{\rho \sigma}
$$

\footnotetext{
${ }^{2}$ We disregard nonanalytic functions of $m^{2}$ and the term $R_{\alpha \beta \mu \nu} e^{\alpha \mu} e^{\nu \beta}$ which is redundant due to the cyclic property $R_{\mu \nu \alpha \beta}+R_{\mu \alpha \beta \nu}+R_{\mu \beta \nu \alpha}=0$.
} 
where $b_{j}(j=0,1,2,3)$ are arbitrary constants for now. By manipulating and simplifying as much as possible, we obtain the following expression:

$$
\begin{aligned}
\mathcal{C}= & +\left[b_{3}\left(2 f_{4}+f_{7}\right)-b_{2}\right] R^{\lambda \alpha} \nabla_{\alpha} \nabla^{\rho} e_{\lambda \rho}+2 b_{3}\left(1-f_{3}\right) R_{\rho \lambda \sigma \alpha} \nabla^{\rho} \nabla^{\alpha} e^{\lambda \sigma}+ \\
& -\left[2 b_{2}\left(\frac{1}{4}+a_{1}\right)-b_{3}\left(\frac{1}{2}-2 a_{1}+f_{7}+2 f_{9}\right)\right] R^{\lambda \alpha} \nabla_{\alpha} \nabla^{\rho} e_{\rho \lambda} \\
& +\left[\left(b_{0} R+b_{1} m^{2}\right)\left(\frac{1}{2}+6 a_{1}\right)+2 b_{2}\left(\frac{1}{4}+a_{1}\right) R-b_{3}\left(m^{2}-2 f_{1} R-2 f_{8} R\right)\right] \nabla^{\lambda} \nabla^{\rho} e_{\rho \lambda}+ \\
& -\left[\left(b_{0} R+b_{1} m^{2}\right)\left(\frac{1}{2}+6 a_{1}\right)+2 b_{2}\left(\frac{1}{4}+a_{1}\right) R-b_{3}\left(m^{2}+2 f_{2} R\right)\right] \square e \\
& +\left(b_{2}+b_{3} f_{5}\right) R_{\lambda \rho} \square e^{\lambda \rho}+\left[b_{2}+b_{3} f_{5}+2\left(a_{1}-\frac{1}{4}\right)\left(b_{2}+b_{3}\right)+b_{3}\right] R_{\lambda \rho} \nabla^{\lambda} \nabla^{\rho} e+\mathcal{C}_{1}
\end{aligned}
$$

where $\mathcal{C}_{1}$ contains up to first derivatives of $e_{\rho \sigma}$. The expression (13) has seven terms with second derivatives of $e_{\rho \sigma}$, which must be eliminated in order to become a scalar constraint. In the special case of the FP theory $\left(a_{1}=1 / 4\right)$, the last two terms with second derivatives can only be simultaneously cancelled if $b_{2}=b_{3}=0$. Back in the other terms we need $b_{0} R+m^{2} b_{1}=0$. However in this case we have no constraint whatsoever. This is in agreement with [24] where the authors have chosen Einstein spaces in order to surmount such difficulty. In the general case $a_{1} \neq$ $1 / 4$ we have to find a solution for the system below:

$$
\begin{gathered}
b_{3}\left(2 f_{4}+f_{7}\right)-b_{2}=0, \\
b_{3}\left(1-f_{3}\right)=0 \\
2 b_{2}\left(\frac{1}{4}+a_{1}\right)-b_{3}\left(\frac{1}{2}-2 a_{1}+f_{7}+2 f_{9}\right)=0, \\
\left(b_{0} R+b_{1} m^{2}\right)\left(\frac{1}{2}+6 a_{1}\right)+2 b_{2}\left(\frac{1}{4}+a_{1}\right) R \\
-b_{3}\left(m^{2}-2 f_{1} R-2 f_{8} R\right)=0
\end{gathered}
$$

$$
\begin{gathered}
\left(b_{0} R+b_{1} m^{2}\right)\left(\frac{1}{2}+6 a_{1}\right)+2 b_{2}\left(\frac{1}{4}+a_{1}\right) R \\
-b_{3}\left(m^{2}+2 f_{2} R\right)=0, \\
b_{2}+b_{3} f_{5}=0, \\
2 b_{2}\left(\frac{1}{4}+a_{1}\right)+2 b_{3}\left(\frac{1}{4}+a_{1}+\frac{f_{5}}{2}\right)=0 .
\end{gathered}
$$

Without restrictions in the background, as shown in the Appendix, we have not been able to solve the previous system and get $e=0$ from the scalar constraint. So, we are going to restrict the gravitational background to Einstein spaces $^{3}$ as in the FP case [12,24],

$$
R_{\mu \nu}=\frac{R}{4} g_{\mu \nu}
$$

Now we can rewrite (10), (11) and (13) as follows:

$$
\begin{aligned}
& \mathcal{C}_{\rho} \doteq \nabla^{\sigma} E_{\rho \sigma}=\left(1-2 f_{3}-2 f_{6}\right) R_{\rho \lambda \sigma \alpha} \nabla^{\alpha} e^{\lambda \sigma}+\left(1+2 f_{6}\right) R_{\rho \lambda \sigma \alpha} \nabla^{\alpha} e^{\sigma \lambda}+2 \tilde{f}_{1} R \nabla^{\lambda} e_{\rho \lambda} \\
&+\left[\frac{1}{2}\left(\frac{1}{4}-a_{1}+4 \tilde{f}_{8}\right) R-m^{2}\right] \nabla^{\lambda} e_{\lambda \rho}+\left[\frac{1}{2}\left(\frac{1}{4}+a_{1}+4 \tilde{f}_{2}\right) R+m^{2}\right] \nabla_{\rho} e=0, \\
& \mathcal{C}_{\rho \sigma} \doteq E_{\rho \sigma}-E_{\sigma \rho}=+\left(-2 a_{1}+\frac{1}{2}\right)\left(\nabla_{\rho} \nabla^{\mu} e_{\mu \sigma}-\nabla_{\sigma} \nabla^{\mu} e_{\mu \rho}\right)+2\left(f_{3}+2 f_{6}\right) R_{\rho \beta \sigma \nu}\left(e^{\beta \nu}-e^{\nu \beta}\right) \\
&+\left[m^{2}+\left(2 \tilde{f}_{1}-2 \tilde{f}_{8}\right) R\right]\left(e_{\rho \sigma}-e_{\sigma \rho}\right)=0,
\end{aligned}
$$

\footnotetext{
${ }^{3}$ Altogether with Bianchi identities we have $\nabla^{\mu} R_{\mu \nu \rho \sigma}=0$ and $\nabla^{\mu} R=\partial^{\mu} R=0$.
} 


$$
\begin{aligned}
\mathcal{C} & \doteq \tilde{b}_{1} g^{\rho \sigma} E_{\rho \sigma}+b_{3} \nabla^{\rho} \nabla^{\sigma} E_{\rho \sigma} \\
= & +2 b_{3}\left(1-f_{3}\right) R_{\rho \lambda \sigma \alpha} \nabla^{\rho} \nabla^{\alpha} e^{\lambda \sigma}+\left[-6 \tilde{b}_{1}\left(a_{1}+\frac{1}{12}\right)+\frac{b_{3}}{2}\left(\frac{1}{4}+a_{1}+4 \tilde{f}_{2}\right) R+b_{3} m^{2}\right] \square e \\
& +\left[6 \tilde{b}_{1}\left(a_{1}+\frac{1}{12}\right)+\frac{b_{3}}{2}\left(\frac{1}{4}-a_{1}+4 \tilde{f}_{1}+4 \tilde{f}_{8}\right) R-b_{3} m^{2}\right] \nabla^{\lambda} \nabla^{\rho} e_{\rho \lambda} \\
& +\tilde{b}_{1}\left[3 m^{2}+\left(2 \tilde{f}_{1}+8 \tilde{f}_{2}+\frac{f_{3}}{2}+2 \tilde{f}_{8}\right) R\right] e=0 .
\end{aligned}
$$

Motivated by the substitution of (21) back in (8) and (12) we have defined:

$$
\begin{aligned}
& \tilde{f}_{1} \doteq f_{1}+\frac{f_{4}}{4}+\frac{f_{9}}{4}, \\
& \tilde{f}_{2} \doteq f_{2}+\frac{f_{5}}{4}, \\
& \tilde{f}_{8} \doteq f_{8}+\frac{f_{7}}{4}, \\
& \tilde{b}_{1} \doteq b_{0} R+b_{1} m^{2}+\frac{b_{2} R}{4} .
\end{aligned}
$$

The expression (22) is already a vector constraint since it does not have second derivatives of the field. It corresponds to four constraints, in total. The same does not occur in expressions (23) and (24). First, let us turn (24) into a scalar constraint. We need to solve the system below:

$$
\begin{aligned}
b_{3}\left(1-f_{3}\right) & =0, \\
-6 \tilde{b}_{1}\left(a_{1}+\frac{1}{12}\right)+\frac{b_{3}}{2}\left(\frac{1}{4}+a_{1}+4 \tilde{f}_{2}\right) R+b_{3} m^{2} & =0, \\
6 \tilde{b}_{1}\left(a_{1}+\frac{1}{12}\right)+\frac{b_{3}}{2}\left(\frac{1}{4}-a_{1}+4 \tilde{f}_{1}+4 \tilde{f}_{8}\right) R-b_{3} m^{2} & =0 .
\end{aligned}
$$

It is easy to see that the solution of (26) back in (24) leads to the scalar constraint $e=0$, provided the coefficient of $e$ is different from zero in (24). However, the expression (23) still has terms with second derivatives. For these terms to be cancelled, ${ }^{4}$ it is necessary that $\nabla^{\mu} e_{\mu \nu}=0$. We can get this from the vector constraint (22) if an appropriate choice of parameters is made. More specifically, since the solution of (26) requires $f_{3}=1$, if we set $\tilde{f}_{1}=0$ and $f_{6}=-\frac{1}{2}$, we obtain automatically from (22) that $\nabla^{\mu} e_{\mu \nu}=0$ as far as the coefficient of $\nabla^{\mu} e_{\mu \nu}$ in (22) is non-null. The solution of the system given in (26) with the additional equations $\tilde{f}_{1}=0$ and $f_{6}=-\frac{1}{2}$ is given by

\footnotetext{
${ }^{4}$ If $a_{1}=\frac{1}{4}$, those terms would be eliminated, but this specific value for $a_{1}$ represents the FP case and it is not of our interest here.
}

$$
\begin{aligned}
& f_{3}=1 \\
& \tilde{f}_{8}=-\frac{1}{8}-\tilde{f}_{2} \\
& \tilde{b}_{1}=\frac{b_{3}}{1+12 a_{1}}\left[2 m^{2}+\left(\frac{1}{4}+a_{1}+4 \tilde{f}_{2}\right) R\right] .
\end{aligned}
$$

Returning this solution in (22), (23) and (24) we finally get all necessary constraints. More specifically, from (24) we obtain the scalar constraint:

$$
e=0
$$

Using (27) and the result $e=0$ in (22), we have the vector constraint:

$$
\nabla^{\sigma} e_{\sigma \rho}=0
$$

Finally, using (27) and the results (28) and (29) in (23), we achieve the tensor constraint:

$$
e_{[\rho \sigma]}=0
$$

once its coefficient in (23) is nonvanishing too. Summarizing, we have found all the FP constraints:

$$
\begin{aligned}
e & =0, \\
\nabla^{\sigma} e_{\sigma \rho} & =0, \\
e_{[\rho \sigma]} & =0,
\end{aligned}
$$

if the restrictions below are respected

$$
\begin{gathered}
b_{3} \tilde{m}^{2}\left[2 \tilde{m}^{2}+\left(-\frac{1}{4}+a_{1}\right) R\right]\left[3 \tilde{m}^{2}-\frac{R}{2}\right] \neq 0, \\
\tilde{m}^{2} \equiv m^{2}+\left(\frac{1}{4}+2 \tilde{f}_{2}\right) R
\end{gathered}
$$

while the equations of motion become

$$
E_{\rho \sigma}=\left(\square-\tilde{m}^{2}\right) e_{\rho \sigma}+2 R_{\rho \alpha \sigma \beta} e^{\alpha \beta} .
$$

Therefore, we end up with $16-11=5$ degrees of freedom, which is the correct count for a massive spin-2 
particle $(5=2 s+1)$. The final curved space theory still contains 2 free parameters: $\tilde{f}_{2}$ and $a_{1}$, with the restriction (34) and $\left(a_{1}+1 / 12\right)\left(a_{1}-1 / 4\right) \neq 0$.

For the sake of comparison with [21] we focus now on a special subcase of Einstein spaces, namely the maximally symmetric spaces:

$$
R_{\alpha \beta \rho \sigma}=\frac{R}{12}\left(g_{\alpha \rho} g_{\beta \sigma}-g_{\alpha \sigma} g_{\beta \rho}\right) \text {. }
$$

All the results from the previous section can be brought consistently to the maximally symmetric spaces (37). Using (25) and (27), the Lagrangian (8) in a maximally symmetric background becomes

$$
\begin{aligned}
\mathcal{L}^{(\mathrm{MSS})}\left(a_{1}\right)= & -\frac{1}{4} \nabla^{\mu} e^{\alpha \beta} \nabla_{\mu} e_{\alpha \beta}-\frac{1}{4} \nabla^{\mu} e^{\alpha \beta} \nabla_{\mu} e_{\beta \alpha}+a_{1} \nabla^{\alpha} e_{\alpha \beta} \nabla_{\mu} e^{\mu \beta}+\frac{1}{2} \nabla^{\alpha} e_{\alpha \beta} \nabla_{\mu} e^{\beta \mu} \\
& +\frac{1}{4} \nabla^{\alpha} e_{\beta \alpha} \nabla_{\mu} e^{\beta \mu}+\left(a_{1}+\frac{1}{4}\right) \nabla^{\mu} e \nabla_{\mu} e-\left(a_{1}+\frac{1}{4}\right) \nabla^{\mu} e\left(\nabla^{\alpha} e_{\alpha \mu}+\nabla^{\alpha} e_{\mu \alpha}\right)+ \\
& -\frac{m^{2}}{2}\left(e_{\alpha \beta} e^{\beta \alpha}-e^{2}\right)-\frac{1}{24} \operatorname{Re}^{\alpha \beta} e_{\alpha \beta}+\left(\tilde{f}_{2}+\frac{1}{12}\right) R e^{2}+-\frac{1}{4}\left(\frac{11}{12}+a_{1}+4 \tilde{f}_{2}\right) R e^{\alpha \beta} e_{\beta \alpha} .
\end{aligned}
$$

On the other hand, it has been presented in [21] a model for massive spin-2 particles also with a nonsymmetric tensor $e_{\mu \nu} \neq e_{\nu \mu}$ minimally coupled to maximally symmetric background.

The Lagrangian is known as dual massive gravity and is given by

$$
\begin{aligned}
\mathcal{L}^{(\text {dual })}= & \frac{1}{2} \nabla_{\rho} e_{\nu \sigma}\left(-\nabla^{\rho} e^{\nu \sigma}-\nabla^{\nu} e^{\rho \sigma}+\nabla^{\nu} e^{\sigma \rho}-\nabla^{\rho} e^{\sigma \nu}\right. \\
& \left.+\nabla^{\sigma} e^{\rho \nu}+\nabla^{\sigma} e^{\nu \rho}\right)-m^{2}\left(e_{\mu \nu} e^{\nu \mu}-e^{2}\right)
\end{aligned}
$$

As already discussed in [20], the model presented in [21] is recovered from $\mathcal{L}^{(\mathrm{MSS})}\left(a_{1}\right)$ in the flat space when $a_{1}=-1 / 4$. However, it is important to notice that the assumption of $a_{1}=-1 / 4$ does not require a maximally symmetric space as we have shown here.

The relation between $\mathcal{L}^{\text {(dual) }}$ and $\mathcal{L}^{(\mathrm{MSS})}\left(a_{1}\right)$ is given by

$$
\begin{aligned}
& \mathcal{L}^{(\mathrm{MSS})}\left(a_{1}=-1 / 4\right) \\
& =\frac{1}{2} \mathcal{L}^{(\text {dual })}-\left[\frac{1}{24}+\tilde{f}_{2}\right] R\left(e^{\alpha \beta} e_{\beta \alpha}-e^{2}\right) .
\end{aligned}
$$

Thus, the model of [21] is a subcase of $\mathcal{L}^{(\mathrm{MSS})}\left(a_{1}=-1 / 4\right)$ where

$$
\tilde{f}_{2}=-\frac{1}{24} \text {. }
$$

With the above value of $\tilde{f}_{2}$, the restrictions (34) lead to two forbidden values for the scalar curvature, namely, $R \neq-6 m^{2}$ and $R \neq 12 m^{2}$. The first value differs by a sign from the restriction obtained in [21] while the second one has not been mentioned. It is important to emphasize, however, that $\tilde{f}_{2}$ is a free parameter in the $\mathcal{L}^{g}\left(a_{1}\right)$ model, so the inequality (34) restricts the possible values of $\tilde{f}_{2}$, not of the curvature $R$. This happens because our original Lagrangian is more general than (39). In MSS there are no forbidden values for the scalar curvature in the $\mathcal{L}^{g}\left(a_{1}\right)$ model for any value of $a_{1}$, including $a_{1}=-1 / 4$.

\section{Local symmetries of $\mathcal{L}^{g}\left(a_{1}\right)$}

In the previous sections, we have found all the constraints of the $\mathcal{L}^{g}\left(a_{1}\right)$ model. The form of (22) and (24) suggests that some local symmetries of $\mathcal{L}^{g}\left(a_{1}\right)$ may exist even in the massive case. For example, if the expression obtained for the vector constraint $\nabla^{\sigma} E_{\rho \sigma}$ given in (22) becomes identically null, instead of a vector constraint we would have four identities $\nabla^{\sigma} E_{\rho \sigma} \equiv 0$ and, consequently, the theory acquires the vector symmetry

$$
\delta e_{\rho \sigma}=\nabla_{\sigma} A_{\rho}
$$

since, up to a surface term,

$$
\begin{aligned}
\delta_{A} S & =\int d^{4} x \frac{\delta S}{\delta e^{\rho \sigma}} \delta e^{\rho \sigma}=\int d^{4} x E_{\rho \sigma} \nabla^{\sigma} A^{\rho} \\
& =-\int d^{4} x\left(\nabla^{\sigma} E_{\rho \sigma}\right) A_{\rho}=0 .
\end{aligned}
$$

In order that $\nabla^{\sigma} E_{\rho \sigma}=0$ holds identically, see (22), we need the conditions: 


$$
\begin{aligned}
f_{3} & =1, \\
\tilde{f}_{1} & =0, \\
\tilde{f}_{8} & =-\frac{1}{8}-\tilde{f}_{2}, \\
f_{6} & =-\frac{1}{2}, \\
R & =-\frac{8 m^{2}}{1+4 a_{1}+16 \tilde{f}_{2}} .
\end{aligned}
$$

We have checked explicitly that (42) is indeed a symmetry of $\mathcal{L}^{g}\left(a_{1}\right)$ if we use (44).

Analogously, we could find scalar symmetries for $\mathcal{L}^{g}\left(a_{1}\right)$. The action is invariant under the transformation

$$
\delta e_{\rho \sigma}{ }^{(1)}=\nabla_{\rho} \nabla_{\sigma} \lambda
$$

where $\lambda$ is an arbitrary scalar, provided the conditions below are satisfied:

$$
\begin{aligned}
& f_{3}=1, \\
& \tilde{f}_{8}=-\frac{1}{8}-\tilde{f}_{1}-\tilde{f}_{2}, \\
& R=-\frac{8 m^{2}}{1+4 a_{1}+16 \tilde{f}_{2}} .
\end{aligned}
$$

In addition, there is another possible scalar symmetry which is

$$
\delta e_{\rho \sigma}^{(2)}=12 \nabla_{\rho} \nabla_{\sigma} \lambda+R g_{\rho \sigma} \lambda
$$

where $\lambda$ is an arbitrary scalar and the conditions below are required:

$$
\begin{aligned}
f_{3} & =1, \\
\tilde{f}_{8} & =-\frac{1}{8}-\tilde{f}_{1}-\tilde{f}_{2}, \\
R & =-\frac{12 m^{2}}{1+24 \tilde{f}_{2}} .
\end{aligned}
$$

We leave for a future work a detailed study of the special cases (44), (46) and (48). The appearance of vector and scalar symmetries are usually connected with massless and partially massless theories, respectively.

\section{FAMILY OF LAGRANGIANS $\mathcal{L}_{\mathrm{nFP}}(c)$}

\section{A. Main results in the flat space}

Analogously to the $\mathcal{L}\left(a_{1}\right)$ case, in [22] we can find another family of second order Lagrangians $\mathcal{L}_{\mathrm{nFP}}(c)$ which describes massive spin-2 particles via a nonsymmetric rank-2 tensor in $D=4$ flat spaces ${ }^{5}$

\footnotetext{
${ }^{5}$ The model $\mathcal{L}_{\mathrm{nFP}}(c)$ can be generalized to arbitrary $D \geq 3$; see [22].
}

$$
\begin{aligned}
\mathcal{L}_{\mathrm{nFP}}(c)= & -\frac{1}{2} \partial^{\mu} e^{(\alpha \beta)} \partial_{\mu} e_{(\alpha \beta)}+\frac{1}{6} \partial_{\mu} e\left[\partial^{\mu} e-2 \partial_{\nu} e^{(\nu \mu)}\right] \\
& +\left[\partial^{\alpha} e_{(\alpha \beta)}\right]^{2}-\frac{1}{3}\left[\partial_{\mu} e^{\mu \nu}\right]^{2}-\frac{m^{2}}{2}\left(e_{\mu \nu} e^{\nu \mu}+c e^{2}\right) .
\end{aligned}
$$

The real constant $c$ is arbitrary and nFP stands for "nonFierz-Pauli" since we do not need to have $c=-1$. In such a special case, however, the model $\mathcal{L}_{\mathrm{nFP}}(c=-1)$ coincides with $\mathcal{L}\left(a_{1}\right)$ at $a_{1}=-\frac{1}{12}$. The massless case $\mathcal{L}_{\mathrm{nFP}}^{m=0}(c)$ first appeared in [25] and describes massless spin-2 particles. If $c \neq-\frac{1}{4}$ the FP conditions can be derived from the equations of motion as follows:

$$
\begin{aligned}
E_{\mu \nu}= & \square e_{(\mu \nu)}+\frac{\eta_{\mu \nu}}{3}\left(\partial^{\alpha} \partial^{\beta} e_{\alpha \beta}-\square e\right)+\frac{1}{3} \partial_{\mu} \partial_{\nu} e-\partial_{\mu} \partial^{\alpha} e_{(\nu \alpha)}+ \\
& -\partial_{\nu} \partial^{\alpha} e_{(\alpha \mu)}+\frac{2}{3} \partial_{\mu} \partial^{\alpha} e_{\alpha \nu}-m^{2}\left(e_{\nu \mu}+c \eta_{\mu \nu} e\right)=0
\end{aligned}
$$

From $\partial^{\nu} E_{\mu \nu}=0$, we have

$$
\partial^{\nu} e_{\nu \mu}+c \partial_{\mu} e=0
$$

Back in (50), we obtain from $E_{\mu \nu}-E_{\nu \mu}=0$ :

$$
e_{[\mu \nu]}=0 .
$$

From $\eta^{\mu \nu} E_{\mu \nu}=0$, we have

$$
m^{2}\left(c+\frac{1}{4}\right) e=0 \Rightarrow e=0
$$

and, consequently, from (51) now we have

$$
\partial^{\alpha} e_{\alpha \nu}=0 .
$$

Thus, the equations of motion given in (50) become the Klein-Gordon equations:

$$
\left(\square-m^{2}\right) e_{(\mu \nu)}=0 .
$$

If $c=-\frac{1}{4}$ the model $\mathcal{L}_{\mathrm{nFP}}(c)$ is invariant under Weyl transformations: $\delta_{W} e_{\mu \nu}=\eta_{\mu \nu} \phi$. We can fix the gauge $e=0$ and obtain all the FP conditions (52), (53) and (54) and the Klein-Gordon equations (55).

\section{B. Generalization of $\mathcal{L}_{\mathrm{nFP}}(c)$ to curved spaces}

\section{General setup and constraints}

For $\mathcal{L}_{\mathrm{nFP}}(c)$ the procedure was analogous to that used for $\mathcal{L}\left(a_{1}\right)$. The most general expression for $\mathcal{L}_{\mathrm{nFP}}(c)$ is the following one: 


$$
\begin{aligned}
\mathcal{L}_{\mathrm{nFP}}^{g}(c)= & -\frac{1}{4} \nabla^{\mu} e^{\alpha \beta} \nabla_{\mu} e_{\alpha \beta}-\frac{1}{4} \nabla^{\mu} e^{\alpha \beta} \nabla_{\mu} e_{\beta \alpha}-\frac{1}{12} \nabla^{\alpha} e_{\alpha \beta} \nabla_{\lambda} e^{\lambda \beta}+\frac{1}{2} \nabla^{\alpha} e_{\alpha \beta} \nabla_{\lambda} e^{\beta \lambda} \\
& +\frac{1}{4} \nabla^{\alpha} e_{\beta \alpha} \nabla_{\lambda} e^{\beta \lambda}+\frac{1}{6} \nabla^{\mu} \nabla_{\mu} e-\frac{1}{3} \nabla^{\alpha} e_{\alpha \beta} \nabla^{\beta} e-\frac{m^{2}}{2}\left(e_{\alpha \beta} e^{\beta \alpha}+c e^{2}\right) \\
& +d_{1} R e^{\alpha \beta} e_{\alpha \beta}+d_{2} R e^{2}+d_{3} R_{\alpha \beta \mu \nu} e^{\alpha \mu} e^{\beta \nu}+d_{4} R_{\alpha \beta} e^{\alpha \mu} e^{\beta}{ }_{\mu}+d_{5} R_{\alpha \beta} e^{\alpha \beta} e \\
& +d_{6} R_{\alpha \beta \mu \nu} e^{\alpha \beta} e^{\mu \nu}+d_{7} R_{\alpha \beta} e^{\alpha \mu} e_{\mu}{ }^{\beta}+d_{8} R e^{\alpha \beta} e_{\beta \alpha}+d_{9} R_{\alpha \beta} e^{\mu \alpha} e_{\mu}{ }^{\beta}
\end{aligned}
$$

where $d_{j}(j=1,2, \ldots, 9)$ are arbitrary constants for now. The equations of motion are

$$
\begin{aligned}
E_{\rho \sigma} \doteq \frac{\delta S_{\mathrm{nFP}}^{g}(c)}{\delta e^{\rho \sigma}}= & \frac{1}{2} \square\left(e_{\rho \sigma}+e_{\sigma \rho}\right)+\frac{1}{6} \nabla_{\rho} \nabla^{\lambda} e_{\lambda \sigma}-\frac{1}{2}\left(\nabla_{\rho} \nabla^{\lambda} e_{\sigma \lambda}+\nabla_{\sigma} \nabla^{\lambda} e_{\rho \lambda}\right)-\frac{1}{2} \nabla_{\sigma} \nabla^{\alpha} e_{\alpha \rho}+ \\
& -\frac{1}{3} g_{\rho \sigma} \square e+\frac{1}{3} \nabla_{\rho} \nabla_{\sigma} e+\frac{1}{3} g_{\rho \sigma} \nabla^{\beta} \nabla^{\alpha} e_{\alpha \beta}-m^{2}\left(e_{\sigma \rho}+c e g_{\sigma \rho}\right)+2 d_{1} R e_{\rho \sigma} \\
& +2 d_{2} \operatorname{Re}_{\rho \sigma}+2 d_{3} R_{\rho \beta \sigma \nu} e^{\beta \nu}+2 d_{4} R_{\rho \beta} e^{\beta}{ }_{\sigma}+d_{5} R_{\rho \sigma} e+d_{5} g_{\rho \sigma} R^{\alpha \beta} e_{\alpha \beta} \\
& +2 d_{6} R_{\alpha \beta \rho \sigma} e^{\alpha \beta}+d_{7} R^{\alpha}{ }_{\sigma} e_{\alpha \rho}+d_{7} R_{\rho}{ }^{\alpha} e_{\sigma \alpha}+2 d_{8} R_{\sigma \rho}+2 d_{9} R_{\sigma}{ }^{\beta} e_{\rho \beta}=0 .
\end{aligned}
$$

The vector constraint is the following expression

$$
\begin{aligned}
\mathcal{C}_{\rho} \doteq \nabla^{\sigma} E_{\rho \sigma}= & \left(1-2 d_{3}-2 d_{6}\right) R_{\rho \lambda \sigma \alpha} \nabla^{\alpha} e^{\lambda \sigma}+\left(1+2 d_{6}\right) R_{\rho \lambda \sigma \alpha} \nabla^{\alpha} e^{\sigma \lambda}+d_{5} R_{\alpha \beta} \nabla_{\rho} e^{\alpha \beta} \\
& +\left(\frac{1}{2}-2 d_{3}-2 d_{6}+d_{7}\right) e^{\lambda \sigma} \nabla_{\lambda} R_{\sigma \rho}+\left(-1+2 d_{3}+d_{5}\right) e^{\lambda \sigma} \nabla_{\rho} R_{\sigma \lambda} \\
& +\left(\frac{1}{2}+2 d_{4}+2 d_{6}\right) e^{\sigma \lambda} \nabla_{\lambda} R_{\sigma \rho}+\left(\frac{1}{2}+2 d_{9}\right) R^{\lambda \mu} \nabla_{\mu} e_{\rho \lambda}+2 d_{1} R \nabla^{\sigma} e_{\rho \sigma} \\
& +\left(\frac{1}{2}+d_{7}\right) R^{\lambda \alpha} \nabla_{\alpha} e_{\lambda \rho}+\left(\frac{1}{6}+d_{7}\right) R_{\lambda \rho} \nabla_{\mu} e^{\mu \lambda}+\left(\frac{1}{4}+2 d_{1}+d_{9}\right) e_{\rho \sigma} \nabla^{\sigma} R \\
& +\left(2 d_{4}-\frac{1}{2}\right) R_{\lambda \rho} \nabla_{\mu} e^{\lambda \mu}+\left(\frac{1}{4}+\frac{d_{7}}{2}+2 d_{8}\right) e_{\lambda \rho} \nabla^{\lambda} R+\left(2 d_{8} R-m^{2}\right) \nabla^{\sigma} e_{\sigma \rho} \\
& +\left(\frac{1}{3}+d_{5}\right) R_{\alpha \rho} \nabla^{\alpha} e+\left(2 d_{2} R-m^{2} c\right) \nabla_{\rho} e+\left(2 d_{2}+\frac{d_{5}}{2}\right) e \nabla_{\rho} R=0 .
\end{aligned}
$$

The tensor constraint will be obtained from the expression below:

$$
\begin{aligned}
\mathcal{C}_{\rho \sigma} \doteq E_{\rho \sigma}-E_{\sigma \rho}= & \frac{2}{3}\left(\nabla_{\rho} \nabla^{\lambda} e_{\lambda \sigma}-\nabla_{\sigma} \nabla^{\lambda} e_{\lambda \rho}\right)+\left(m^{2}+2\left(d_{1}-d_{8}\right) R\right)\left(e_{\rho \sigma}-e_{\sigma \rho}\right) \\
& +2\left(2 d_{6}+d_{3}\right) R_{\rho \sigma \alpha \beta} e^{\alpha \beta}+\left(2 d_{4}-d_{7}\right)\left(R_{\rho}^{\beta} e_{\beta \sigma}-R_{\sigma}^{\beta} e_{\beta \rho}\right)+\left(d_{7}-2 d_{9}\right)\left(R_{\rho}^{\beta} e_{\sigma \beta}-R_{\sigma}^{\beta} e_{\rho \beta}\right)=0 .
\end{aligned}
$$

Regarding the scalar constraint, due to the Weyl symmetry of the kinetic terms in $\mathcal{L}_{\mathrm{nFP}}^{g}(c)$ we do not need to add second derivatives of equations of motion in order to produce a constraint as in (24). We can simply have

$$
\begin{aligned}
\mathcal{C} & \doteq g^{\rho \sigma} E_{\rho \sigma} \\
& =\left[-m^{2}(1+4 c)+\left(2 d_{1}+8 d_{2}+d_{5}+2 d_{8}\right) R\right] e+2\left(d_{3}+d_{4}+2 d_{5}+d_{7}+d_{9}\right) R_{\sigma \beta} e^{\sigma \beta}=0 .
\end{aligned}
$$

Thus, we have a scalar constraint in arbitrary gravitational backgrounds. In the Appendix we show that although it is possible to get $e=0$ from (60) in arbitrary backgrounds, we are not able to have a curved space version of the tensor constraint without restricting the background space. Henceforth we assume Einstein spaces (21). Let us rewrite (58), (59) and (60) as follows: 


$$
\begin{gathered}
\mathcal{C}_{\rho} \doteq \nabla^{\sigma} E_{\rho \sigma}=\left(1-2 d_{3}-2 d_{6}\right) R_{\rho \lambda \sigma \mu} \nabla^{\mu} e^{\lambda \sigma}+\left(1+2 d_{6}\right) R_{\rho \lambda \sigma \mu} \nabla^{\mu} e^{\sigma \lambda}+\tilde{d}_{1} R \nabla^{\sigma} e_{\rho \sigma} \\
+\left[\left(\frac{1}{6}+2 \tilde{d}_{8}\right) R-m^{2}\right] \nabla^{\sigma} e_{\sigma \rho}+\left[\left(\frac{1}{12}+2 \tilde{d}_{2}\right) R-m^{2} c\right] \nabla_{\rho} e=0 \\
\mathcal{C}_{\rho \sigma} \doteq E_{\rho \sigma}-E_{\sigma \rho}=\frac{2}{3}\left(\nabla_{\rho} \nabla^{\lambda} e_{\lambda \sigma}-\nabla_{\sigma} \nabla^{\lambda} e_{\lambda \rho}\right)+2\left(d_{3}+2 d_{6}\right) R_{\rho \sigma \alpha \beta} e^{\alpha \beta}++\left[m^{2}+\left(2 \tilde{d}_{1}-2 \tilde{d}_{8}+\frac{d_{9}}{2}\right) R\right]\left(e_{\rho \sigma}-e_{\sigma \rho}\right)=0 \\
\mathcal{C} \doteq g^{\rho \sigma} E_{\rho \sigma}=\left[-m^{2}(1+4 c)+\left(2 \tilde{d}_{1}+8 \tilde{d}_{2}+\frac{d_{3}}{2}+2 \tilde{d}_{8}\right) R\right] e=0
\end{gathered}
$$

where we have defined

$$
\begin{aligned}
& \tilde{d}_{1} \doteq d_{1}+\frac{d_{4}}{4}+\frac{d_{9}}{4}, \\
& \tilde{d}_{2} \doteq d_{2}+\frac{d_{5}}{4}, \\
& \tilde{d}_{8} \doteq d_{8}+\frac{d_{7}}{4} .
\end{aligned}
$$

Therefore, (63) leads to the scalar constraint $e=0$, provided the coefficient of $e$ is different from zero. On the other hand, (62) still has terms with second derivatives. In order to solve this, we need $\nabla^{\mu} e_{\mu \nu}=0$. We can get this automatically from the vector constraint (61) by setting

$$
\tilde{d}_{1}=0, \quad d_{3}=1, \quad d_{6}=-\frac{1}{2}
$$

as far as the coefficient of $\nabla^{\mu} e_{\mu \nu}$ does not vanish.

Back in the tensor constraint (62) we obtain $e_{[\mu \nu]}=0$ as far as its coefficient is nonvanishing too. In summary, all 11 Fierz-Pauli constraints (31), (32) and (33) are confirmed if

$$
\tilde{m}^{2}\left(\tilde{m}^{2}-\frac{R}{6}\right)\left\{(1+4 c) \tilde{m}^{2}+\left[8\left(c \tilde{d}_{8}-\tilde{d}_{2}\right)-\frac{1}{2}\right] R\right\} \neq 0
$$

$$
\tilde{m}^{2} \equiv m^{2}-2 \tilde{d}_{8} R
$$

while the equations of motion become

$$
E_{\rho \sigma}=\left(\square-\tilde{m}^{2}\right) e_{\rho \sigma}+2 R_{\rho \alpha \sigma \beta} e^{\alpha \beta}
$$

where the free paramenters $\tilde{d}_{2}$ and $\tilde{d}_{8}$ must satisfy the conditions (66).

\section{Local symmetries of $\mathcal{L}_{\mathrm{nFP}}^{g}(c)$}

The model $\mathcal{L}_{\mathrm{nFP}}^{g}(c)$ also presents vector and scalar symmetries. There is one vector symmetry which comes from the transformation

$$
\delta e_{\rho \sigma}=\nabla_{\sigma} A_{\rho}
$$

where $A_{\rho}$ is an arbitrary vector. We need a nonvanishing scalar curvature $(R \neq 0)$ and the following conditions:

$$
\begin{aligned}
\tilde{d}_{1} & =0 ; \quad d_{6}=-\frac{1}{2} ; \quad d_{3}=1 ; \\
\tilde{m}^{2} & =\frac{R}{6} \\
\tilde{d}_{2} & =-\frac{1}{24}+\frac{c}{12}+\tilde{d}_{8} c .
\end{aligned}
$$

Such conditions imply that the vector constraint (61) be identically null, i.e., $\nabla^{\sigma} E_{\rho \sigma} \equiv 0$.

On the other hand, starting from the general scalar transformation

$$
\delta e_{\rho \sigma}=A_{1} \nabla_{\rho} \nabla_{\sigma} \lambda+A_{2} g_{\rho \sigma} \lambda+A_{3} g_{\rho \sigma} \square \lambda
$$

where $\lambda$ is an arbitrary field and $A_{j}(j=1,2,3)$ are arbitrary constants, we have found three scalar symmetries for $\mathcal{L}_{\mathrm{nFP}}^{g}(c)$.

The first one is

$$
\delta e_{\rho \sigma}{ }^{(1)}=g_{\rho \sigma} \lambda
$$

where we must have

$$
(1+4 c) \tilde{m}^{2}=R\left[\frac{1}{2}+8\left(\tilde{d}_{2}-c \tilde{d}_{8}\right)\right] .
$$

The second scalar symmetry is 


$$
\delta e_{\rho \sigma}^{(2)}=-4 \nabla_{\rho} \nabla_{\sigma} \lambda+g_{\rho \sigma} \square \lambda
$$

where the relations below must hold:

$$
\begin{aligned}
d_{3} & =1, \\
\tilde{m}^{2} & =\left(1+12 \tilde{d}_{1}\right) \frac{R}{6} .
\end{aligned}
$$

Finally, the last scalar symmetry is given by

$$
\delta e_{\rho \sigma}{ }^{(3)}=\nabla_{\rho} \nabla_{\sigma} \lambda
$$

where the conditions below must be obeyed:

$$
\begin{aligned}
d_{3} & =1 \\
\tilde{d}_{2} & =-\frac{1}{24}+\frac{c}{12}+c\left(\tilde{d}_{1}+\tilde{d}_{8}\right), \\
\tilde{m}^{2} & =\left(1+12 \tilde{d}_{1}\right) \frac{R}{6} .
\end{aligned}
$$

The symmetry (76) and conditions (77) follow from the previous scalar symmetries (72) and (74).

\section{CONCLUSION}

Here we have studied massive spin-2 models via a nonsymmetric rank-2 tensor in a curved background. As in the usual Fierz-Pauli (FP) case with a symmetric tensor, nonminimal couplings are necessary. The work here is a preliminary one and parallels the work [24] on the FP model. As in that case we have assumed that the Ansätze (8) and (56) are linear on curvatures and their coefficients are analytic functions of $m^{2}$. Although there seems to be slightly more freedom now in choosing the background metric than in the FP case, we have selected, for simplicity, background spaces of the Einstein type as in [24]. We have succeeded in finding nontrivial solutions for the coefficients of our Ansätze by getting rid of second derivatives in the tensor, vector, and scalar constraints. In particular, we have generalized from maximally symmetric spaces to Einstein spaces a previous work in the literature [21] carried out for a massive spin-2 theory with nonsymmetric tensor $e_{\mu \nu} \neq e_{\nu \mu}$, which corresponds to the model $\mathcal{L}\left(a_{1}\right)$ of Sec. II at the specific point $a_{1}=-1 / 4$. Regarding the model $\mathcal{L}_{n F P}(c)$, due to the Weyl symmetry of the kinetic terms, the scalar constraint has easily led to the traceless condition $e=0$ but now the problem has moved to the tensor constraint and once again we have found it convenient to choose Einstein spaces.

Comparing the results obtained here in Einstein spaces for $\mathcal{L}\left(a_{1}\right)$ and $\mathcal{L}_{n F P}(c)$ with the ones obtained in [24] for the usual FP model, the main difference is that, besides the scalar and vector constraint, we now have a tensor constraint $C_{\rho \mu}=0$; see (11) and (23). However, due to the vector constraint $\nabla^{\mu} e_{\mu \nu}=0$, the constraint $C_{\rho \mu}=0$ amounts to $e_{\mu \nu}-e_{\nu \mu}=0$ without further restriction in the background. So there seems to be no fundamental difference to the usual FP case in curved space. Since in the FP case there is no restriction on the background metric when we allow the coefficients to be nonanalytic functions of $\mathrm{m}^{2}$, we would like to address that point also in the case of our nonsymmetric models. This is under investigation now.

Moreover, in both cases of $\mathcal{L}\left(a_{1}\right)$ and $\mathcal{L}_{n F P}(c)$, we believe that less restrictive conditions on the coefficients can be obtained by getting rid of second order derivatives of time only; this is under study. We are also analyzing the special points in the parameters space where the local symmetries mentioned in the previous sections show up. They indicate massless and partially massless theories even if $m^{2} \neq 0$. The truly massless cases $m=0$ in both $\mathcal{L}\left(a_{1}\right)$ and $\mathcal{L}_{n F P}(c)$ theories are also worth investigating in curved space. Especially in the second case where, at least in the flat space, we have massless spin-2 particles just like in the massless version of the FP model.

As a final comment, we notice that the ghost free massive gravity theories, see [8] and [9], accommodate massive gravitons propagating in any gravitational background, see [26-28]. Those results agree with earlier perturbative (in powers of $1 / \mathrm{m}^{2}$ ) calculations. Thus, if we obtain correct Lagrangian constraints for the models discussed here, in the case of nonanalytic coefficients, we would be prompted to search for nonlinear (self-interacting) versions of $\mathcal{L}\left(a_{1}\right)$ and $\mathcal{L}_{n F P}(c)$.

\section{ACKNOWLEDGMENTS}

H. G. M. F. thanks FAPESP (2013/25368-0) and D. D. thanks CNPq (307278/2013-1) for financial support.

\section{APPENDIX: CONSTRAINTS IN A GENERAL BACKGROUND}

\section{1. $\mathcal{L}\left(a_{1}\right)$}

We are looking for a solution of the system (14)-(20) without imposing restrictions on the background space. Since $f_{i}(i=1, \ldots, 9)$ and $b_{j}(j=0, \ldots, 3)$ are constants, we demand that the coefficients of $R$ in Eqs. (17) and (18) are null, i.e.,

$$
\begin{aligned}
b_{0}\left(\frac{1}{2}+6 a_{1}\right)+2 b_{2}\left(\frac{1}{4}+a_{1}\right)+2 b_{3}\left(f_{1}+f_{8}\right) & =0, \\
b_{0}\left(\frac{1}{2}+6 a_{1}\right)+2 b_{2}\left(\frac{1}{4}+a_{1}\right)-2 b_{3} f_{2} & =0 .
\end{aligned}
$$

The solution, singular at $a_{1}=1 / 4$ as expected, is given by 
$f_{3}=1, \quad f_{4}=\frac{1}{2}+f_{9}, \quad f_{5}=\frac{\left(1+4 a_{1}\right)}{\left(-1+4 a_{1}\right)}, \quad f_{7}=-\frac{8 a_{1}}{\left(-1+4 a_{1}\right)}-2 f_{9}, \quad f_{2}=-\frac{1+8 a_{1}\left(1+2 a_{1}\right)}{4\left(-1+4 a_{1}\right)}+\frac{b_{0}}{2 b_{1}}$,
$f_{1}=\frac{1+8 a_{1}\left(1+2 a_{1}\right)}{4\left(-1+4 a_{1}\right)}-\frac{b_{0}}{2 b_{1}}-f_{8}, \quad b_{3}=\frac{1}{2} b_{1}\left(1+12 a_{1}\right), \quad b_{2}=-\frac{b_{1}\left(1+12 a_{1}\right)\left(1+4 a_{1}\right)}{2\left(-1+4 a_{1}\right)}$.

Plugging this solution back into (13), we obtain a scalar constraint in a general background:

$$
\begin{aligned}
\mathcal{C}=\mathcal{C}_{1}= & \frac{\left(1+12 a_{1}\right)}{\left(-1+4 a_{1}\right)}\left\{-\frac{b_{1}\left(1+4 a_{1}\right)^{2}}{2\left(-1+4 a_{1}\right)} R^{\rho \sigma} R_{\rho \sigma} e+\frac{b_{1}\left(1-12 a_{1}\right)}{2} \nabla^{\lambda} R^{\sigma \rho} \nabla_{\rho} e_{\lambda \sigma}\right. \\
& \left.+4 b_{1} a_{1} e_{\lambda \sigma} \square R^{\lambda \sigma}+\frac{b_{1}\left(1+12 a_{1}\right)}{2\left(-1+4 a_{1}\right)} \nabla_{\rho} R^{\lambda \sigma} \nabla^{\rho} e_{\lambda \sigma}-\frac{b_{1}\left(1+4 a_{1}\right)}{2} \nabla^{\sigma} R^{\lambda \alpha} \nabla_{\alpha} e_{\lambda \sigma}\right\} \\
& +\frac{b_{1}\left(1+12 a_{1}\right)}{2}\left\{\left[-\frac{b_{0}}{b_{1}}+\frac{1+8 a_{1}\left(1+2 a_{1}\right)}{4\left(-1+4 a_{1}\right)}\right] \nabla^{\lambda} R \nabla^{\rho} e_{\rho \lambda}+\right. \\
& \left.-\left[\frac{b_{0}}{b_{1}}+\frac{1-2 a_{1}\left(-1+4 a_{1}\right)}{-1+4 a_{1}}-2 f_{9}\right] \nabla^{\lambda} R \nabla^{\rho} e_{\lambda \rho}\right\} \\
& +\frac{\left(1+12 a_{1}\right)}{\left(-1+4 a_{1}\right)}\left\{\left(b_{0} R+b_{1} m^{2}\right)+\frac{b_{1}\left(1+4 a_{1}\right)}{2}\left[m^{2}+\frac{b_{0} R}{b_{1}}-\frac{\left[16 a_{1}\left(1+a_{1}\right)+3\right] R}{2\left(-1+4 a_{1}\right)}\right]\right\} R^{\rho \sigma} e_{\rho \sigma} \\
& +b_{1}\left(1+12 a_{1}\right) R^{\rho \sigma} R_{\rho \beta \sigma \nu} e^{\beta \nu}+\frac{\left(1+12 a_{1}\right)}{\left(-1+4 a_{1}\right)}\left[\frac{b_{1}\left(1+12 a_{1}\right)\left(1+4 a_{1}\right)}{2\left(-1+4 a_{1}\right)}-8 b_{1} a_{1}\right] R^{\rho \sigma} R_{\rho \beta} e^{\beta}{ }_{\sigma} \\
& +\frac{b_{1}\left(1+12 a_{1}\right)}{2}\left\{\left[\frac{2 b_{0}}{b_{1}}-\frac{1+16 a_{1}\left(1+3 a_{1}\right)}{4\left(-1+4 a_{1}\right)}\right] \nabla_{\lambda} R \nabla^{\lambda} e+\left[\frac{b_{0}}{b_{1}}-\frac{2 a_{1}\left(1+4 a_{1}\right)}{\left(-1+4 a_{1}\right)}\right](\square R) e\right\} \\
& +\left\{\left(b_{0} R+b_{1} m^{2}\right)\left[3 m^{2}-\frac{1+16 a_{1}\left(1+3 a_{1}\right)}{2\left(-1+4 a_{1}\right)} R+\frac{3 b_{0} R}{b_{1}}\right]+\right. \\
& \left.-\frac{b_{1}\left(1+12 a_{1}\right)\left(1+4 a_{1}\right)}{2\left(-1+4 a_{1}\right)}\left[m^{2}+\frac{b_{0} R}{b_{1}}-\frac{\left[1+8 a_{1}\left(1+2 a_{1}\right)\right] R}{2\left(-1+4 a_{1}\right)}\right] R\right\} e .
\end{aligned}
$$

We have not been able to derive $e=0$ from (A4) without restrictions on the background space.

\section{2. $\mathcal{L}_{n F P}$}

For arbitrary backgrounds if we choose $d_{9}=-d_{3}-d_{4}-2 d_{5}-d_{7}$ and $d_{2}=-\left(2 d_{1}+d_{5}+2 d_{8}\right) / 8$ and assume $c \neq-1 / 4$, the scalar constraint (60) becomes simply $e=0$. Putting those results back into (58) and (59), we obtain

$$
\begin{aligned}
\mathcal{C}_{\rho}= & \left(1-2 d_{3}-2 d_{6}\right) R_{\rho \lambda \sigma \alpha} \nabla^{\alpha} e^{\lambda \sigma}+\left(1+2 d_{6}\right) R_{\rho \lambda \sigma \alpha} \nabla^{\alpha} e^{\sigma \lambda}+d_{5} R_{\alpha \beta} \nabla_{\rho} e^{\alpha \beta} \\
& +\left(\frac{1}{2}-2 d_{3}-2 d_{6}+d_{7}\right) e^{\lambda \sigma} \nabla_{\lambda} R_{\sigma \rho}+\left(-1+2 d_{3}+d_{5}\right) e^{\lambda \sigma} \nabla_{\rho} R_{\sigma \lambda} \\
& +\left(\frac{1}{2}+2 d_{4}+2 d_{6}\right) e^{\sigma \lambda} \nabla_{\lambda} R_{\sigma \rho}+\left(\frac{1}{2}-2 d_{3}-2 d_{4}-4 d_{5}-2 d_{7}\right) R^{\lambda \mu} \nabla_{\mu} e_{\rho \lambda} \\
& +2 d_{1} R \nabla^{\sigma} e_{\rho \sigma}+\left(\frac{1}{4}+2 d_{1}-d_{3}-d_{4}-2 d_{5}-d_{7}\right) e_{\rho \sigma} \nabla^{\sigma} R \\
& +\left(2 d_{4}-\frac{1}{2}\right) R_{\lambda \rho} \nabla_{\mu} e^{\lambda \mu}+\left(\frac{1}{4}+\frac{d_{7}}{2}+2 d_{8}\right) e_{\lambda \rho} \nabla^{\lambda} R+\left(2 d_{8} R-m^{2}\right) \nabla^{\sigma} e_{\sigma \rho} \\
& +\left(\frac{1}{2}+d_{7}\right) R^{\lambda \alpha} \nabla_{\alpha} e_{\lambda \rho}+\left(\frac{1}{6}+d_{7}\right) R_{\lambda \rho} \nabla_{\mu} e^{\mu \lambda}
\end{aligned}
$$




$$
\begin{aligned}
\mathcal{C}_{\rho \sigma}= & \frac{2}{3}\left(\nabla_{\rho} \nabla^{\lambda} e_{\lambda \sigma}-\nabla_{\sigma} \nabla^{\lambda} e_{\lambda \rho}\right)+\left(m^{2}+2\left(d_{1}-d_{8}\right) R\right)\left(e_{\rho \sigma}-e_{\sigma \rho}\right) \\
& +2\left(2 d_{6}+d_{3}\right) R_{\rho \sigma \alpha \beta} e^{\alpha \beta}+\left(2 d_{4}-d_{7}\right)\left(R_{\rho}^{\beta} e_{\beta \sigma}-R_{\sigma}^{\beta} e_{\beta \rho}\right) \\
& +\left(2 d_{3}+2 d_{4}+4 d_{5}+3 d_{7}\right)\left(R_{\rho}^{\beta} e_{\sigma \beta}-R_{\sigma}^{\beta} e_{\rho \beta}\right)=0 .
\end{aligned}
$$

There are still second derivatives in $\mathcal{C}_{\rho \sigma}$. We could think of determining $\nabla^{\sigma} e_{\sigma \rho}$ as a function of the remaining terms in (A5) at $d_{8}=0$ and plugging it back in (A6) which leads to

$$
\begin{aligned}
\mathcal{C}_{\rho \sigma}= & \frac{4}{3 m^{2}}\left[\left(1-2 d_{3}-2 d_{6}\right) R_{[\sigma \lambda \beta \mu} \nabla_{\rho]} \nabla^{\mu} e^{\lambda \beta}+\left(1+2 d_{6}\right) R_{[\sigma \lambda \beta \mu} \nabla_{\rho]} \nabla^{\mu} e^{\beta \lambda}\right. \\
& +\left(\frac{1}{2}-2 d_{3}-2 d_{4}-4 d_{5}-2 d_{7}\right) R^{\lambda \mu} \nabla_{[\rho} \nabla_{\mu} e_{\sigma] \lambda}+\left(\frac{1}{2}+d_{7}\right) R^{\lambda \mu} \nabla_{[\rho} \nabla_{\mu} e_{\lambda \sigma]} \\
& +\left(\frac{1}{6}+d_{7}\right) R_{\mu[\sigma} \nabla_{\rho]} \nabla_{\lambda} e^{\lambda \mu}+\left(-\frac{1}{2}+2 d_{4}\right) R_{\mu[\sigma} \nabla_{\rho]} \nabla_{\lambda} e^{\mu \lambda}+d_{5} R_{\alpha \beta} \nabla_{[\rho} \nabla_{\sigma]} e^{\alpha \beta} \\
& \left.+2 d_{1} R \nabla_{[\rho} \nabla^{\beta} e_{\sigma] \beta}+\nabla_{[\rho} \mathcal{F}_{\sigma]}\right]+\left(m^{2}+2\left(d_{1}-d_{8}\right) R\right)\left(e_{\rho \sigma}-e_{\sigma \rho}\right) \\
& +2\left(2 d_{6}+d_{3}\right) R_{\rho \sigma \alpha \beta} e^{\alpha \beta}+\left(2 d_{4}-d_{7}\right)\left(R_{\rho}^{\beta} e_{\beta \sigma}-R_{\sigma}^{\beta} e_{\beta \rho}\right) \\
& +\left(2 d_{3}+2 d_{4}+4 d_{5}+3 d_{7}\right)\left(R_{\rho}^{\beta} e_{\sigma \beta}-R_{\sigma}^{\beta} e_{\rho \beta}\right)
\end{aligned}
$$

where $\mathcal{F}_{\alpha}$ does not contain any derivative of $e_{\rho \sigma}$ and is defined as follows:

$$
\begin{aligned}
\mathcal{F}_{\alpha} \doteq & +\left(\frac{1}{2}-2 d_{3}-2 d_{6}+d_{7}\right) e^{\lambda \mu} \nabla_{\lambda} R_{\mu \alpha}+\left(-1+2 d_{3}+d_{5}\right) e^{\lambda \mu} \nabla_{\alpha} R_{\mu \lambda} \\
& +\left(\frac{1}{2}+2 d_{4}+2 d_{6}\right) e^{\mu \lambda} \nabla_{\lambda} R_{\mu \alpha}+\left(\frac{1}{4}+2 d_{1}-d_{3}-d_{4}-2 d_{5}-d_{7}\right) e_{\alpha \lambda} \nabla^{\lambda} R+\left(\frac{1}{4}+\frac{d_{7}}{2}\right) e_{\lambda \alpha} \nabla^{\lambda} R .
\end{aligned}
$$

Unfortunately, without any restriction on the background space we have not been able to avoid second derivatives of $e_{\mu \nu}$ in the tensor constraint (A7). Therefore, just like the $\mathcal{L}^{g}\left(a_{1}\right)$ case, we are led to Einstein spaces $R_{\mu \nu}=\frac{R}{4} g_{\mu \nu}$ once again.

[1] A. G. Riess et al., Observational evidence from supernovae for an accelerating universe and a cosmological constant, Astron. J. 116, 1009 (1998).

[2] S. Perlmutter et al., Measurements of Omega and Lambda from 42 high redshift supernovae, Astrophys. J. 517, 565 (1999).

[3] LIGO Scientific Collaboration and Virgo Collaboration, Observation of Gravitational Waves from a Binary Black Hole Merger, Phys. Rev. Lett. 116, 061102, 2016.

[4] LIGO Scientific Collaboration and Virgo Collaboration, Tests of General Relativity with GW150914, Phys. Rev. Lett. 116, 221101, 2016.

[5] H. van Dam and M. J. G. Veltman, Massive and massless Yang-Mills and gravitational fields, Nucl. Phys. B22, 397 (1970).

[6] V. I. Zakharov, Linearized gravitation theory and the graviton mass, Pisma Zh. Eksp. Teor. Fiz. 12, 447 (1970) [JETP Lett. 12, 312 (1970)].
[7] D. G. Boulware and S. Deser, Inconsistency of finite range gravitation, Phys. Lett. 40B, 227 (1972).

[8] C. de Rham, G. Gabadadze, and A. J. Tolley, Resummation of Massive Gravity, Phys. Rev. Lett. 106, 231101 (2011).

[9] S. Hassan and R. A. Rosen, Bimetric gravity from ghost-free massive gravity, J. High Energy Phys. 02 (2012) 126.

[10] A. I. Vainshtein, To the problem of nonvanishing gravitation mass, Phys. Lett. 39B, 393 (1972).

[11] N. Arkani-Hamed, H. Georgi, and M. D. Schwartz, Effective field theory for massive gravitons and gravity in theory space, Ann. Phys. (Berlin) 305, 96 (2003).

[12] K. Hinterbichler, Theoretical aspects of massive gravity, Rev. Mod. Phys. 84, 671 (2012).

[13] C. de Rham, Massive Gravity, Living Rev. Relativ. 17 (2014) 7.

[14] C. Aragone and S. Deser, Constraints on gravitationally coupled tensor fields, Nuovo Cimento A 3, 709 (1971).

[15] C. Aragone and S. Deser, Consistency problems of spin-2 gravity coupling, Nuovo Cimento Soc. Ital. Fis. 57, 33 (1980). 
[16] I. Buchbinder, D. Gitman, V. Krykhtin, and V. Pershin, Equations of motion for massive spin 2 field coupled to gravity, Nucl. Phys. B584, 615 (2000).

[17] G. Velo and D. Zwanziger, Noncausality and other defects of interaction Lagrangians for particles with spin one and higher, Phys. Rev. 188, 2218 (1969).

[18] G. Velo, Anomalous behavior of a massive spin two charged particle in an external electromagnetic field, Nucl. Phys. B43, 389 (1972).

[19] M. Fierz and W. Pauli, On relativistic wave equations for particles of arbitrary spin in an electromagnetic field, Proc. R. Soc. A 173, 211 (1939).

[20] D. Dalmazi, Massive spin-2 particle from a rank-2 tensor, Phys. Rev. D 87, 125027 (2013).

[21] K. Morand and S. N. Solodukhin, Dual massive gravity, arXiv:1204.6224; Phys. Lett. B 715B, 260 (2012).

[22] D. Dalmazi, A. L. R. dos Santos, and E. L. Mendonça, Massive spin-2 theories in arbitrary $D \geq 3$ dimensions, Ann. Phys. (Amsterdam) 354, 385 (2015).
[23] A. Koenigstein, F. Giacosa, and D. H. Rischke, Classical and quantum theory of the massive spin-two field, Ann. Phys. (Amsterdam) 368, 16 (2016).

[24] I. L. Buchbinder, V. A. Krykhtin, and V. D. Pershin, On consistent equations for massive spin-2 field coupled to gravity in string theory, Phys. Lett. B 466, 216 (1999).

[25] H. Casini, R. Montemayor, and L. F. Urrutia, Duality for symmetric second rank tensors. 2. The linearized gravitational field, Phys. Rev. D 68, 065011 (2003).

[26] L. Bernard, C. Deffayet, and M. von Strauss, Consistent massive graviton on arbitrary backgrounds, Phys. Rev. D 91, 104013 (2015).

[27] L. Bernard, C. Deffayet, and M. von Strauss, Massive graviton on arbitrary background: derivation, syzygies, applications, J. Cosmol. Astropart. Phys. 06 (2015) 038.

[28] L. Bernard, C. Deffayet, A. Schmidt-May, and M. von Strauss, Linear spin-2 fields in most general backgrounds, Phys. Rev. D 93, 084020 (2016). 